\title{
Downregulation of microRNA-221 decreases migration and invasion in fibroblast-like synoviocytes in rheumatoid arthritis
}

\author{
SHUZHONG YANG and YOUGENG YANG
}

\author{
Department of Orthopaedics, The Second Hospital of Jilin University, Changchun, Jilin 130041, P.R. China
}

Received July 13, 2014; Accepted March 24, 2015

DOI: $10.3892 / \mathrm{mmr} .2015 .3642$

\begin{abstract}
MicroRNAs (miRNAs/miRs) are a group of non-coding RNAs that regulate the activity of target mRNAs and cellular processes, and which have been implicated in the pathogenesis of autoimmune diseases. miR-221 is one of the miRNAs that regulate cell proliferation, invasion and apoptosis in tumors. However, the role of miR-221 in rheumatoid arthritis (RA) remains to be fully elucidated. Therefore, the present study was undertaken to identify the role of miR-221 in RA. The expression of miR-221 in serum and synovial tissues of patients with RA and healthy controls was confirmed by reverse transcription quantitative polymerase chain reaction analysis. The effects of miR-221 on pro-inflammatory cytokines and a chemokine were assessed by ELISA. The effects of miR-221 on cell apoptosis, migration and invasion in fibroblast-like synoviocytes (FLS) were also assessed in vitro. The results showed that miR-221 expression in serum and synovial tissues of patients with RA was higher than that in healthy controls. Downregulation of miR-221 significantly suppressed the expression of pro-inflammatory cytokines and the chemokine, and inhibited FLS cell migration and invasion via inhibiting vascular endothelial growth factor, matrix metalloproteinase (MMP)-3 and MMP-9 expression. In addition, downregulation of miR-221 significantly induced cell apoptosis and decreased survivin and X-linked inhibitor of apoptosis protein expression. These findings indicated that downregulation of miR-221 inhibited the expression of pro-inflammatory cytokines and the chemokine, suppressed FLS cell migration and invasion, and induced cell apoptosis. Therefore, miR-221 is likely to be implicated in RA pathogenesis via these mechanisms, and may be a target for the treatment of RA.
\end{abstract}

Correspondence to: Professor Yougeng Yang, Department of Orthopaedics, The Second Hospital of Jilin University, 218 Ziqiang Street, Changchun, Jilin 130041, P.R. China

E-mail: yygeng1479@126.com

Key words: microRNAs, miR-221, rheumatoid arthritis, migration, invasion, inflammatory cytokine

\section{Introduction}

Rheumatoid arthritis (RA) is a systemic, chronic inflammatory disease characterized by synovial hyperplasia, joint destruction and extraarticular manifestations, and it has a significant impact on morbidity as well as mortality, leading to hyperproliferation of synovial cells and tissue destruction $(1,2)$. Although it is thought that the interaction of genetic, immunological and environmental factors contributes to the disease (3), the pathogenesis of RA, in particular the role of microRNAs (miRNAs/miRs) in RA, has remained to be fully elucidated.

miRNAs are endogenous small (21-25 nucleotides), single-stranded, non-coding RNAs that mediate mRNA cleavage, translational repression and mRNA destabilization (4). To date, using various computational and experimental approaches, $>2,200$ human miRNAs have been registered in the miR Base database (release 19) (5). It has been reported that alterations in miRNA expression levels impact expression of the target genes in a 1.2-fold to 4-fold manner and thus represent a fine-tuning mechanism regulating protein expression (6). Therefore, miRNAs are thought to have a crucial role in regulating innate as well as adaptive immune responses (7). Dysregulation of miRNA in peripheral blood mononuclear cells (PBMCs), T lymphocytes, synovial fibroblasts and osteoclasts, each of those considered a key effector of joint destruction, has been shown to cause inflammation, degradation of the extracellular matrix and invasive behavior of resident cells (8-11). Altered expression of circulating miRNAs has repeatedly been reported to be associated with RA $(12,13)$.

miR-221 is one of the miRNAs that is evolutionarily conserved among vertebrates and abundantly expressed in plasma $(14,15)$. Recently, accumulating evidence has demonstrated that miR-221 has a crucial role in cell growth, oncogenesis, invasion, migration and drug resistance in tumor cells (16-18). In addition, miR-221 also regulates cytokine production (19). In particular, a recent study showed that miR-221/222 was dysregulated in synovial fibroblasts in RA (20). However, the role of miR-221 in RA has remained elusive. Therefore, the present study analyzed the association between miR-221 expression and the expression of pro-inflammatory cytokines and a chemokine in fibroblast-like synoviocytes (FLS). The effects of miR-221 on cell apoptosis, migration as well as invasion in FLS were also examined. 


\section{Materials and methods}

Patients and healthy controls. Fresh peripheral blood and synovial tissue specimens were obtained from healthy donors $(n=18)$ with no history of autoimmune diseases or patients with rheumatoid arthritis (RA, $\mathrm{n}=22$ ) at the Department of Orthopedic Surgery, the Second Hospital of Jilin University (Changchun, China). Ethical approval of the use of human samples in this study was granted by the Ethics Committee of Jilin University, and written informed consent was obtained from all study participants. All RA patients fulfilled the American College of Rheumatology criteria for classification of the disease (21).

A 5-ml sample of peripheral venous blood from each study participant was collected using EDTA-coated tubes (BD Vacutainer $5 \mathrm{ml}$; BD Diagnostics, Franklin Lakes, NJ, USA) according to standard procedures. The blood samples were then centrifuged at $1,000 \mathrm{xg}$ for $10 \mathrm{~min}$ at $4^{\circ} \mathrm{C}$, the serum was immediately separated from the blood, and RNA was immediately extracted.

Cell culture. FLS were isolated from synovial tissues by enzymatic digestion as previously described (22). FLS were grown in Dulbecco's modified Eagle's medium (DMEM; Gibco-BRL, Invitrogen Life Technologies, Carlsbad, CA, USA) medium containing $10 \%$ heat-inactivated fetal calf serum (FCS; Invitrogen Life Technologies) supplemented with antibiotics $(100 \mathrm{mg} / \mathrm{ml}$ streptomycin and $100 \mathrm{U} / \mathrm{ml}$ penicillin; Sigma-Aldrich, St. Louis, MO, USA) in a humidified incubator at $37^{\circ} \mathrm{C}$ in $5 \% \mathrm{CO}_{2}$. Cultures of FLS were subjected to experimental procedures at passages 4-6.

Cell transfection. miR-221 inhibitors and corresponding negative controls (NC) were purchased from Ambion (Life Technologies, Thermo Fisher Scientific, Waltham, MA, USA). The Oligofectamine ${ }^{\mathrm{TM}}$ Transfection Reagent from Invitrogen Life Technologies was used for cell transfection according to the manufacturer's instructions. The final concentration of the miRNA inhibitor was $50 \mathrm{nM}$.

miRNA reverse transcription quantitative polymerase chain reaction (RT- $q P C R)$ analysis. Total RNA of cells, serum or tissue, including miRNAs, was extracted using the Qiagen miRNeasy mini kit (catalogue no. 217004; Qiagen, Hilden, Germany) according to the manufacturer's instructions. The purity and concentration of RNA were determined by using a dual-beam ultraviolet spectrophotometer (BioSpectrometer plus; Eppendorf, Hamburg, Germany). RNA was then reversely transcribed into cDNA using an NCode VILO miRNA cDNA Synthesis kit (Life Technologies). Real-time qPCR was performed using Express SYBR Green ER qPCR Supermix (both from Life Technologies) and an Applied Biosystems 7500 Thermocycler (Life Technologies). For detection of miR-221 and U6 small nuclear RNA expression, specific primers were obtained from Exiqon (Vedbaek, Denmark), i.e. U6 snRNA PCR primer set (product no. 203907), and $\mathrm{LNA}^{\mathrm{TM}}$ Homo sapiens-miR-221 PCR primer set (product no. 204532). The qPCR conditions were as follows: An initial $95^{\circ} \mathrm{C}$ for 4 min followed by 40 cycles of $95^{\circ} \mathrm{C}$ for $10 \mathrm{sec}$ and $58^{\circ} \mathrm{C}$ for $30 \mathrm{sec}$. A dissociation curve was established after each PCR in order to verify amplification specificity. The integrity of the miRNA and the efficiency of qPCR in each sample were confirmed by the endogenous control U6 small nuclear RNA. Negative control experiments were set without cDNA template. The data were analyzed with SDS Relative Quantification Software version 2.06 (Life Technologies).

ELISA. To quantify tumor necrosis factor (TNF)- $\alpha$, interleukin (IL)-6, IL-1 $\beta$ and chemokine (C-X-C motif) ligand 16 (CXCL16) production in FLS, cells transfected with miR-221 inhibitor and corresponding negative control, respectively, were cultured for $8 \mathrm{~h}$, followed by stimulation with $10 \mu \mathrm{g} / \mathrm{ml}$ lipopolysaccharide (LPS; Sigma-Aldrich), and then incubated for $24 \mathrm{~h}$. Twenty-four hours after LPS stimulation, culture supernatants were harvested, and the concentrations of TNF- $\alpha$, IL-6, IL- $1 \beta$ and CXCL16 were measured using an ELISA kit for human TNF- $\alpha$, IL-6, IL-1 $\beta$ and CXCL16 (Genzyme, Cambridge, MA, USA) in a microplate reader (Multiskan MK3; Thermo Fisher Scientific). The concentrations of each were normalized relative to the total number of cells.

Apoptosis analysis. The terminal deoxynucleotidyl transferase dUTP nick end labeling (TUNEL) assay was performed to determine the number of apoptotic cells. In brief, cellular DNA fragmentation was measured with the ApoTag Red in situ Apoptosis detection kit (Chemicon International, EMD Millipore, Billerica, MA, USA) according to the manufacturer's instructions after FLS cells were transfected with miR-221 inhibitor and corresponding negative control for $24 \mathrm{~h}$ and exposed to LPS for $48 \mathrm{~h}$. In order to quantify the apoptotic cells, the TUNEL-positive cells were counted using a confocal microscope (IX51; Olympus, Tokyo, Japan). The number of apoptotic cells in five random high-power fields were counted and expressed as a percentage of total cells (apoptotic fraction).

In addition, survivin and $\mathrm{X}$-linked inhibitor of apoptosis protein (XIAP) were also detected by western blot as an additional indicator of apoptosis.

Cell migration and invasion assay. To assess the effect of miR-221 on FLS cell migration, a wound-healing assay was performed. In brief, FLS cells were seeded at a density of $5 \times 10^{3}$ cells/well in a 96-well plate after FLS were pre-treated with miR-221 inhibitor or corresponding negative control (NC) for $24 \mathrm{~h}$ and exposed to LPS for $48 \mathrm{~h}$. After the cells had formed a fluent monolayer, they were observed under a fluorescent microscope (X41; Olympus). A linear scratch was formed using a 10- $\mu 1$ pipette tip at $48 \mathrm{~h}$ after transfection. Wounded monolayers were washed with phosphate-buffered saline (PBS) to remove detached cells and debris. Photomicrographs of ten random fields were obtained (original magnification, $x 100$ ), and cells were counted to calculate the average number of cells that had migrated.

For the in vitro invasion assay, Transwell chamber experiments were performed using inserts coated using a Matrigel basement membrane matrix (Matrigel; Becton Dickinson, La Jolla, CA, USA). In brief, the Matrigel was diluted in serum-free cold media, placed into upper chambers of a 
A

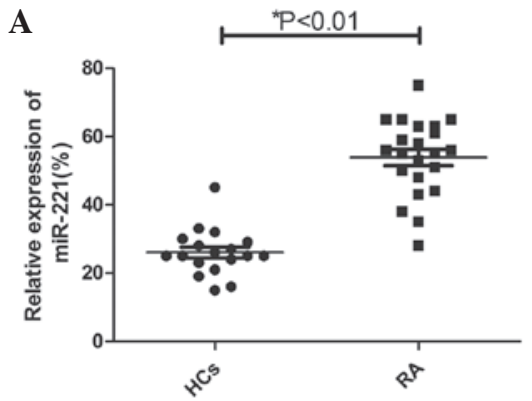

C

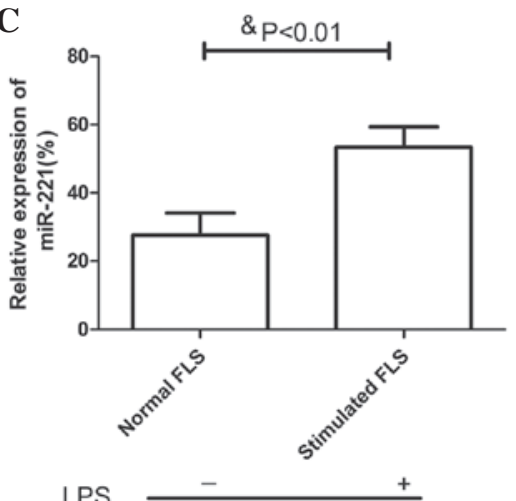

B

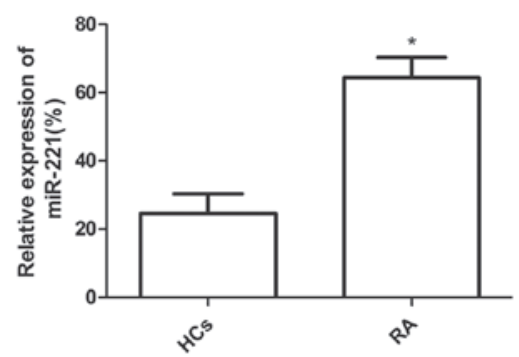

$\mathbf{D}$

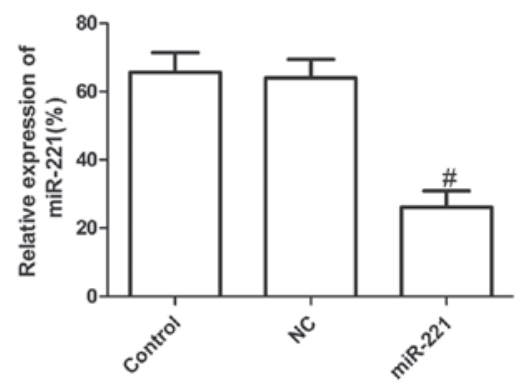

Figure 1. miR-221 expression is significantly increased in serum and synovial tissues from patients with RA. Expression of miR-221 in (A) serum and (B) synovial tissues of $18 \mathrm{HCs}$ and in the 22 patients with RA was measured by qPCR. (C) After stimulation with LPS, miR-221 mRNA levels of FLS cells were evaluated by qPCR. (D) After transfection with miR-221 inhibitor and corresponding NC, miR-221 mRNA levels in FLS cells stimulated with LPS were determined by qPCR. Values are expressed as the mean \pm standard deviation. ${ }^{*} \mathrm{P}<0.01 \mathrm{vs}$. $\mathrm{HCs},{ }^{{ }^{\circ}} \mathrm{P}<0.01$ vs. Normal, ${ }^{\text {}} \mathrm{P}<0.01$ vs. NC. NC, negative control; RA, rheumatoid arthritis; miR, microRNA; FLS, fibroblast-like synoviocytes; qPCR, quantitative polymerase chain reaction; HC, healthy control; LPS, lipopolysaccharide.

24-well Transwell plate and incubated at $37^{\circ} \mathrm{C}$ for $1 \mathrm{~h}$. Cells were re-suspended with serum-free DMEM media at a density of $5 \times 10^{4}$ cells/well and incubated for $48 \mathrm{~h}$ to evaluate cell invasion. Cell invasion was observed using an immunofluorescence microscope (X41; Olympus) by counting the cells that had invaded into the bottom of the cell culture insert. All experiments were performed in triplicate.

Measurement of VEGF production. VEGF production was determined by competitive ELISA. In brief, FLS cells were treated with miR-221 inhibitor and corresponding negative control for $24 \mathrm{~h}$ in 12-well plates and subsequently exposed to LPS for $48 \mathrm{~h}$. These culture media were centrifuged to remove cell debris. Cell-free culture media were collected at $8 \mathrm{~h}$. Then PGE2 levels were measured using Human VEGF ELISA kits (Yanyu, Shanghai, China) according to the manufacturer's instructions.

Western blot analysis. For western blot analyses, FLS cells were harvested and rinsed once with ice-cold PBS and lysed in ice-cold cell lysis buffer (Walterson, London, UK) containing complete protease inhibitor cocktail (Sigma-Aldrich) following pre-treatment of FLS with miR-221 inhibitor or corresponding negative control (NC) for $24 \mathrm{~h}$ and exposure to LPS for $48 \mathrm{~h}$. The protein concentration was determined using the bicinchoninic protein assay kit (KeyGen Biotech, Nanjing, China) using a c-globulin standard curve. Equal amounts of protein $(20 \mu \mathrm{g} / \mathrm{lane})$ from the cell lysates were separated using 10-15\% SDS-PAGE (Sigma-Aldrich) and transferred onto nitrocellulose membranes (Santa Cruz Biotechnology, Inc., Dallas, TX, USA). The membrane was incubated for $2 \mathrm{~h}$ in PBS plus 0.1\% Tween-20 (Sigma-Aldrich) and 5\% non-fat skimmed milk to block non-specific binding. The membranes were then incubated overnight at $4^{\circ} \mathrm{C}$ with the following antibodies: Rabbit monoclonal anti-survivin (1:1,000; cat. no. EP2880Y; Abcam,Cambridge, UK); rabbit polyclonal anti-XIAP (1:1,000; cat. no. 2042s; Cell Signaling Technology, Beverly, MA, USA); mouse monoclonal anti-MMP-3 (1:1,000; cat. no. 69543; EMD Millipore) and mouse monoclonal anti-MMP-9 (1:5,000; cat. no. sc-12759; Santa Cruz Biotechnology, Inc.). Mouse monoclonal anti-GAPDH (1:10,000; cat. no. sc-47724; Santa Cruz Biotechnology, Inc.) was used as a loading control. The membranes were incubated with the appropriate horseradish peroxidase-conjugated immunoglobulin $\mathrm{G}$ (anti-rabbit 1:10,000; cat. no. 7074S; Cell Signaling Technology; or anti-mouse 1:10,000; cat. no. sc-2005; Santa Cruz Biotechnology, Inc.) for $2 \mathrm{~h}$ at room temperature, and protein bands were visualized using an Enhanced Chemiluminescence reagent (Thermo Fisher Scientific).

Statistical analysis. Values are expressed as the mean \pm standard deviation of data from at least three independent experiments. Student's $t$-test or analysis of variance were used. All data were analyzed using the SPSS ${ }^{\circledR}$ statistical package, version 16.0 (SPSS Inc., Chicago, IL, USA) and GraphPad Prism version 5.01 (GraphPad Software, La Jolla, CA, USA) for Windows ${ }^{\circledR}$. $\mathrm{P}<0.05$ was considered to indicate a statistically significant difference between values. 
A

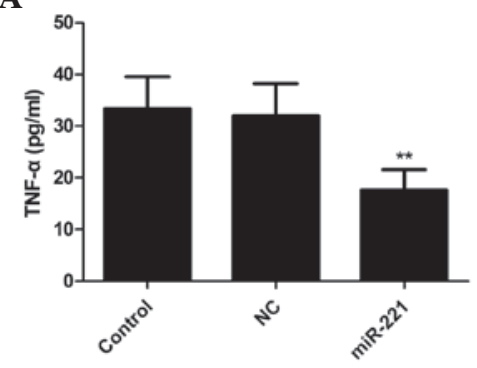

C

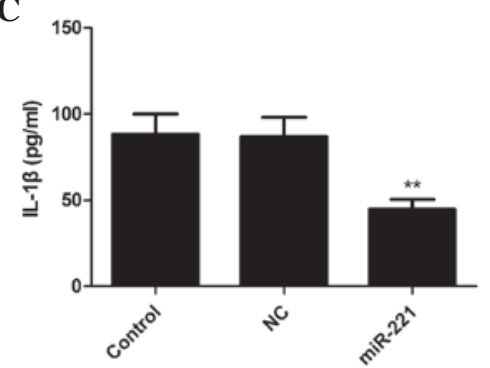

B

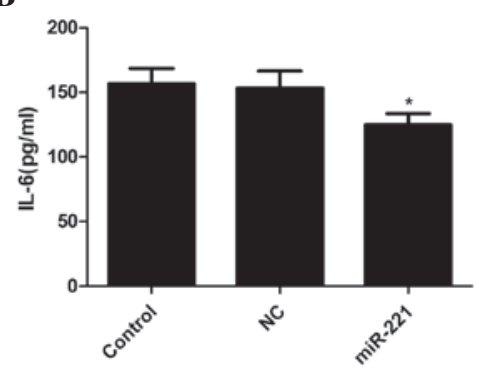

D

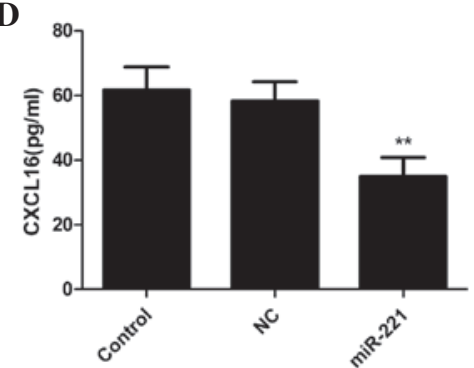

Figure 2. Suppression of LPS-induced expression of pro-inflammatory cytokines in FLS by miR-221 inhibitor. Cells were transfected with miR-221 or corresponding NC and then stimulated with $10 \mathrm{mg} / \mathrm{ml}$ LPS. $24 \mathrm{~h}$ later, the culture supernatants were collected and concentrations of (A) TNF- $\alpha$, (B) IL-6, (C) IL-1 $\beta$ and (D) CXCL16 were determined by ELISA. Values are expressed as the mean \pm standard deviation. ${ }^{*} \mathrm{P}<0.05$ and ${ }^{* *} \mathrm{P}<0.01$ vs. control. NC, negative control; miR, microRNA; FLS, fibroblast-like synoviocytes; TNF, tumor necrosis factor; IL, interleukin.; LPS, lipopolysaccharide; CXCL16, chemokine (C-X-C motif) ligand 16.

A

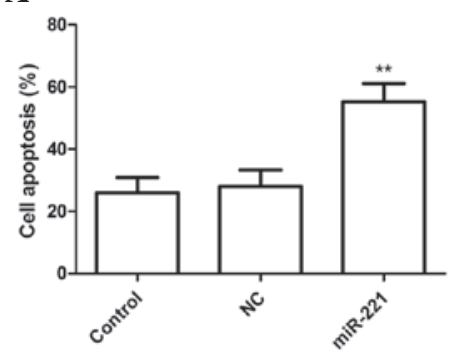

B

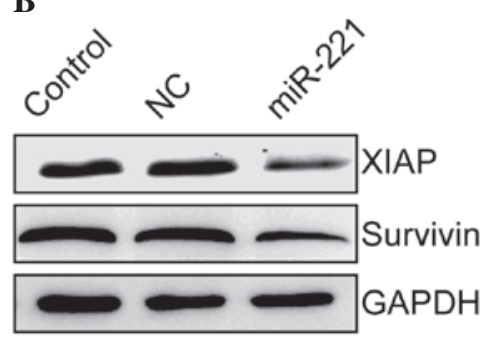

Figure 3. miR-221 inhibitor-induced cell apoptosis in FLS cells stimulated with LPS. (A) FLS cells were pre-treated with miR-221 inhibitor or corresponding $\mathrm{NC}$ for $24 \mathrm{~h}$ and exposed to LPS for $48 \mathrm{~h}$, then cellular apoptosis was measured using the terminal deoxynucleotidyl transferase dUTP nick end labeling assay; (B) FLS cells were pre-treated with miR-221 inhibitor or corresponding NC for $24 \mathrm{~h}$ and exposed to LPS for $48 \mathrm{~h}$, then survivin and XIAP expression were determined by ELISA. Values are expressed as the mean \pm standard deviation. ${ }^{*} \mathrm{P}<0.05$, and ${ }^{* *} \mathrm{P}<0.01$ vs. control. NC, negative control; miR, microRNA; FLS, fibroblast-like synoviocytes; LPS, lipopolysaccharide; XIAP, X-linked inhibitor of apoptosis protein.

\section{Results}

miR-221 expression in serum and synovial tissues is significantly elevated in RA patients. Using RT-qPCR analysis, miR-221 expression levels in serum and synovial tissues of patients with RA and healthy controls were determined. It was found that miR-221 expression in serum and synovial tissues was significantly elevated in RA patients compared with those in healthy controls $(\mathrm{P}<0.01$; Fig. $1 \mathrm{~A}$ and $\mathrm{B})$. In addition, miR-221 expression in human FLS cells was determined. The results showed that miR-221 in FLS cells was significantly upregulated by LPS stimulation compared with that in normal FLS (without stimulation) ( $\mathrm{P}<0.01$; Fig. 1C). To investigate the role of miR-221 in RA, miR-221 inhibitor and corresponding negative control were transfected into FLS cells stimulated with LPS. RT-PCR analysis demonstrated that the expression of miR-221 in cells transfected with miR-221 inhibitor was decreased compared with that in cells transfected with negative control $(\mathrm{P}<0.05$; Fig. 1D).

miR-221 inhibitor decreases LPS-induced pro-inflammatory cytokines and CXCL16 expression in FLS. To examine whether downregulation of miR-221 had any effect on pro-inflammatory cytokines and a chemokine in FLS cells stimulated with LPS, ELISAs were performed to quantify TNF- $\alpha$, IL- 6 , IL- $1 \beta$ and CXCL16 levels. It was found that transfection of miR-221 inhibitor significantly inhibited the expression of TNF- $\alpha$, IL- 6 and IL-1 $\beta$ and CXCL16 upregulated by LPS $(\mathrm{P}<0.01$; Fig. 2A-D). These results suggested that downregulation of miR-221 decreased TNF- $\alpha$, IL- 6 and IL- $1 \beta$ and CXCL16 production in FLS cells stimulated with LPS.

miR-221 induces cell apoptosis of FLS stimulated with LPS. In order to examine whether the miR-221 has any effect on 
A

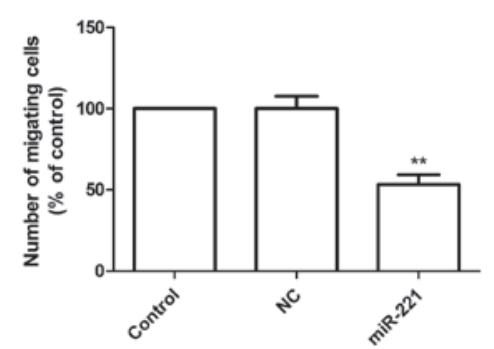

B

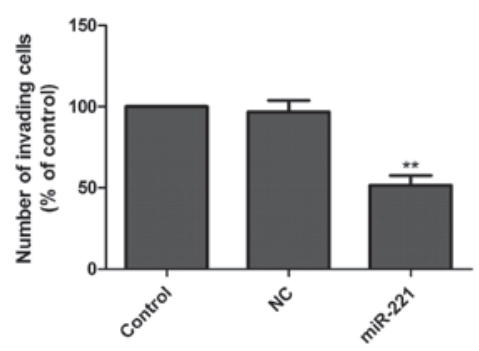

Figure 4. miR-221 inhibitor attenuates the migration and invasion of FLS cells stimulated with LPS. (A) miR-221 inhibitor significantly inhibited the migration activity of FLS cells stimulated by LPS. (B) miR-221 inhibitor significantly reduced the number of invading FLS cells stimulated by LPS. Values are expressed as the mean \pm standard deviation. ${ }^{*} \mathrm{P}<0.05$, and ${ }^{* *} \mathrm{P}<0.01$ vs. control. NC, negative control; miR, microRNA; FLS, fibroblast-like synoviocytes; LPS, lipopolysaccharide.

A

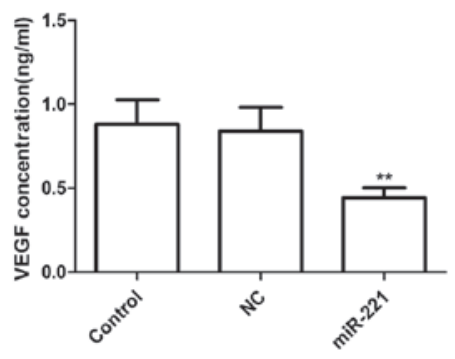

B

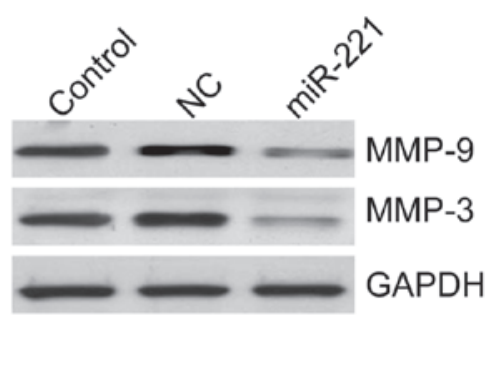

Figure 5. miR-221 inhibitor decreases secretion of VEGF, MMP-3 and MMP-9 by FLS stimulated by LPS. (A) FLS were pre-treated with miR-221 inhibitor or corresponding NC for $24 \mathrm{~h}$ and exposed to human LPS for $48 \mathrm{~h}$, then VEGF production was measured using ELISA. (B) FLS were pre-treated with miR-221 inhibitor or corresponding NC for $24 \mathrm{~h}$ and exposed to human LPS for $48 \mathrm{~h}$, then MMP-3 and MMP-9 protein expression were measured by western blot. Values are expressed as the mean \pm standard deviation. ${ }^{*} \mathrm{P}<0.05$, and ${ }^{* *} \mathrm{P}<0.01$ vs. control. NC, negative control; miR, microRNA; FLS, fibroblast-like synoviocytes; LPS, lipopolysaccharide; VEGF, vascular endothelial growth factor; MMP, matrix metalloproteinase.

cell apoptosis, a TUNEL assay was performed. FLS cells were treated for $24 \mathrm{~h}$ with the miR-221 inhibitor and corresponding negative control and then subsequently exposed to LPS for $48 \mathrm{~h}$, followed by TUNEL assay. The results showed that miR-221 inhibitor markedly increased the percentage of apoptotic cells compared to that in the untreated control group and negative control treatment group $(\mathrm{P}<0.01$; Fig. 3A).

To explore the possible mechanism of the pro-apoptotic effect of miR-221, survivin and XIAP expression were determined by western blot. The results are shown in Fig. 3B, demonstrating that the miR-221 inhibitor significantly decreased survivin and XIAP expression compared with that in the control and negative control treatment groups.

Inhibition of miR-221 reduces FLS migration and invasion of FLS stimulated with LPS. In order to further illustrate the effects of miR-221 on FLS, cell migration and invasion were evaluated. FLS cells stimulated with LPS were transfected with miR-221 inhibitor and corresponding negative control, followed by wound healing and Transwell assays. The results demonstrated that the miR-221 inhibitor significantly attenuated the migration and invasion of FLS $(\mathrm{P}<0.01$; Fig. 4A and B).

Inhibition of miR-221 decreases the expression of VEGF-A, $M M P-1$ and MMP-3 in human RA-FLS. To illustrate the possible underlying mechanism of the effect of miR-221 on the migration and invasion, the effect of miR-221 inhibition on levels of VEGF, MMP-3 and MMP-9 was assessed in FLS stimulated with LPS. The cells were pre-treated with miR-221 inhibitor and corresponding negative control for $24 \mathrm{~h}$ followed by stimulation with LPS for $48 \mathrm{~h}$. VEGF production was measured by ELISA, revealing that VEGF excretion in the supernatant of the miR-221 group was significantly decreased compared with that in the control and negative control groups $(\mathrm{P}<0.05$; Fig. 5A). Finally, the supernatants were assayed for MMP-3 and MMP-9 by western blot analysis. It was found that the miR-221 inhibitor substantially decreased the levels of MMP-3 and MMP-9 in the supernatants compared with those in the controls and negative control group (Fig. 5B). These results may imply that the miR-221 inhibitor attenuated migration and invasion in FLS cells stimulated by LPS via inhibiting VEGF, MMP-3 and MMP-9 expression.

\section{Discussion}

miRNAs are of interest due to their critical role as fine regulators of gene expression at the post-transcriptional level within cells in numerous diseases, which makes them potential targets in the treatment of numerous diseases. For RA accumulating evidence has demonstrated that the expression of miRNAs, including miR-146a, miR-155, miR-16, miR-23b, miR-203, miR-124a, miR-346, miR-223 as well as miR-34a was altered in synovial fibroblasts, peripheral blood mononuclear cells, and T cells from RA patients (8-12,23-29). It has been reported that this abnormal miRNA expression is associated with 
abnormal innate immunity $(23,24)$, inflammation (23), cell proliferation (24) and cell apoptosis (29). Thus, the suppression of overexpressed miRNAs or reconstitution of the expression by restoration of silenced miRNAs is a therapeutic strategy in the treatment of RA (30). Similar to these studies, the present study showed that miR-221 expression in serum and synovial tissues of patients with RA was higher than that in healthy controls, and that downregulation of miR-221 significantly suppressed the expression of pro-inflammatory cytokines and a chemokine, as well as induced cell apoptosis in FLS cells stimulated with LPS.

It has been shown that miR-221 has critical roles in the regulation of cell proliferation, cell cycle, migration and invasion by regulating target genes, and that miR-221 dysregulation is causally involved in the initiation and progression of cancer $(17,18)$. It has also been suggested that miR-221 contributes to $\mathrm{CD} 4^{+} \mathrm{T}$-cell function (31). This indicated that miR-221 is relevant to RA pathogenesis and that it possibly has a crucial role in RA development and progression. To test this hypothesis, the present study analyzed the association between miR-221 expression and pro-inflammatory cytokines and chemokine production in fibroblast-like synoviocytes (FLS). The effects of miR-221 on cell apoptosis, migration as well as invasion in FLS were also determined. The results showed that the miR-221 inhibitor significantly suppressed the expression of pro-inflammatory cytokines and a chemokine, induced cell apoptosis, as well as inhibited migration and invasion in FLS cells stimulated with LPS. These data suggested that miR-221 may be involved in the initiation and progression of RA.

IL-17, TNF- $\alpha$ and IL-1 $\beta$ are important cytokines in the pathogenesis of RA and regulate the cellular immune responses in numerous dimensions, including the generation of T-cell memory, controlling the development and function of regulatory $\mathrm{T}$ cells as well as modulating the secretion of cytokines (32-35). Thus, in the present study, it was also determined whether the expression of miR-221 affected inflammatory conditions in human FLS. The results showed that transfection with miR-221 inhibitor to FLS cells stimulated by LPS inhibited the expression of TNF- $\alpha$, IL- 6 and IL- $1 \beta$, suggesting that the miR-221 inhibitor may have great potential for use as a therapeutic tool in the treatment of RA patients resistant to anti-cytokine therapies.

It is known that synovial cells produce MMP-3 in RA, which has a major role in the cartilage destruction in joints (36). In addition, endogenous MMP-2 or MMP-9 contribute to synovial fibroblast survival, proliferation, migration and invasion in RA (37). The present study showed that an miR-221 inhibitor suppressed VEGF, MMP3 and MMP-9 protein expression, and inhibited migration and invasion in FLS stimulated by LPS. These findings indicated that the observed attenuated FLS migration and invasion by the miR-221 inhibitor may be caused via inhibiting VEGF-A, MMP3 and MMP-9 protein expression.

In conclusion, the findings of the present study provided evidence that miR-221 expression was increased in patients with RA, and that inhibition of miR-221 suppressed pro-inflammatory cytokines, induced cell apoptosis, as well as attenuated migration and invasion via inhibiting VEGF, MMP3 and MMP-9 expression in FLS cells stimulated by LPS. These findings suggested that miR-221 is a potential target in the treatment of RA.

\section{Acknowledgements}

This work was supported by the Science and Technology Research and Innovation Team Fund of Jilin province (no. JL2014054).

\section{References}

1. Feldmann M, Brennan FM and Maini RN: Rheumatoid arthritis. Cell 85: 307-310, 1996.

2. Brennan FM and McInnes IB: Evidence that cytokines play a role in rheumatoid arthritis. J Clin Invest 118: 3537-3545, 2008.

3. McInnes IB and Schett G: The pathogenesis of rheumatoid arthritis. N Engl J Med 365: 2205-2219, 2011.

4. Luo X, Tsai LM, Shen N and Yu D: Evidence for microRNA-mediated regulation in rheumatic diseases. Ann Rheum Dis 69 (Suppl 1): i30-i36, 2010.

5. Kozomara A and Griffiths-Jones S: miR Base: integrating microRNA annotation and deep-sequencing data. Nucleic Acids Res 39: D152-D157, 2011.

6. O'Connell RM, Rao DS and Baltimore D: microRNA regulation of inflammatory responses. Annu Rev Immunol 30: 295-312, 2012.

7. Pauley KM, Cha S and Chan EK: MicroRNA in autoimmunity and autoimmune diseases. J Autoimmun 32: 189-194, 2009.

8. Stanczyk J, Pedrioli DM, Brentano F, et al: Altered expression of MicroRNA in synovial fibroblasts and synovial tissue in rheumatoid arthritis. Arthritis Rheum 58: 1001-1009, 2008.

9. Niimoto T, Nakasa T, Ishikawa M, et al: MicroRNA-146a expresses in interleukin-17 producing $\mathrm{T}$ cells in rheumatoid arthritis patients. BMC Musculoskelet Disord 11: 209, 2010.

10. Stanczyk J, Ospelt C, Karouzakis E, et al: Altered expression of microRNA-203 in rheumatoid arthritis synovial fibroblasts and its role in fibroblast activation. Arthritis Rheum 63: 373-381, 2011.

11. Lu MC, Yu CL, Chen HC, Yu HC, Huang HB and Lai NS: Increased miR-223 expression in T cells from patients with rheumatoid arthritis leads to decreased insulin-like growth factor-1 mediated interleukin-10 production. Clin Exp Immunol 177: 641-51, 2014.

12. Kurowska-Stolarska M, Alivernini S, Ballantine LE, et al: MicroRNA-155 as a proinflammatory regulator in clinical and experimental arthritis. Proc Natl Acad Sci USA 108: 11193-11198, 2011.

13. Nakasa T, Shibuya H, Nagata Y, Niimoto T and Ochi M: The inhibitory effect of microRNA-146a expression on bone destruction in collagen-induced arthritis. Arthritis Rheum 63: 1582-1590, 2011

14. Kawaguchi T, Komatsu S, Ichikawa D, et al: Clinical impact of circulating miR-221 in plasma of patients with pancreatic cancer. Br J Cancer 108: 361-369, 2013.

15. Zhao R, Wu J, Jia W, et al: Plasma miR-221 as a predictive biomarker for chemoresistance in breast cancer patients who previously received neoadjuvant chemotherapy. Onkologie 34: 675-680, 2011.

16. Ergun S, Arman K, Temiz E, et al: Expression patterns of miR-221 and its target caspase-3 in different cancer cell lines. Mol Biol Rep 41: 5877-5881, 2014.

17. Yang X, Yang Y, Gan R, et al: Down-regulation of mir-221 and mir-222 restrain prostate cancer cell proliferation and migration that is partly mediated by activation of SIRT1. PLoS One 9: e98833, 2014.

18. Ye X, Bai W, Zhu H, et al: MiR-221 promotes trastuzumab-resistance and metastasis in HER2-positive breast cancers by targeting PTEN. BMB Rep 47: 268-273, 2014.

19. Brown PN and Yin H: PNA-based microRNA inhibitors elicit anti-inflammatory effects in microglia cells. Chem Commun (Camb) 49: 4415-4417, 2013.

20. Pandis I, Ospelt C, Karagianni N, et al: Identification of microRNA-221/222 and microRNA-323-3p association with rheumatoid arthritis via predictions using the human tumour necrosis factor transgenic mouse model. Ann Rheum Dis 71: 1716-1723, 2012.

21. Aletaha D, Neogi T, Silman AJ, et al: 2010 Rheumatoid arthritis classification criteria: an american college of rheumatology/european league against rheumatism collaborative initiative. Arthritis Rheum 62: 2569-2581, 2010. 
22. Yoshioka Y, Kozawa E, Urakawa H, et al: Suppression of hyaluronan synthesis alleviates inflammatory responses in murine arthritis and in human rheumatoid synovial fibroblasts. Arthritis Rheum 65: 1160-1170, 2013.

23. Semaan N, Frenzel L, Alsaleh G, et al: miR-346 controls release of TNF- $\alpha$ protein and stability of its mRNA in rheumatoid arthritis via tristetraprolin stabilization. PLoS One 6: e19827, 2011

24. Nakamachi Y, Kawano S, Takenokuchi M, et al: MicroRNA-124a is a key regulator of proliferation and monocyte chemoattractant protein 1 secretion in fibroblast-like synoviocytes from patients with rheumatoid arthritis. Arthritis Rheum 60: 1294-1304, 2009.

25. Pauley KM, Satoh M, Chan AL, et al: Upregulated miR-146a expression in peripheral blood mononuclear cells from rheumatoid arthritis patients. Arthritis Res Ther 10: R101, 2008.

26. Zhu S, Pan W, Song X, et al: The microRNA miR-23b suppresses IL-17-associated autoimmune inflammation by targeting TAB2, TAB3 and IKK- $\alpha$. Nat Med 18: 1077-1086, 2012.

27. Nakasa T, Miyaki S, Okubo A, et al: Expression of microRNA-146 in rheumatoid arthritis synovial tissue. Arthritis Rheum 58 1284-1292, 2008.

28. Fulci V, Scappucci G, Sebastiani GD, et al: miR-223 is overexpressed in T-lymphocytes of patients affected by rheumatoid arthritis. Hum Immunol 71: 206-211, 2011.

29. Niederer F, Trenkmann M, Ospelt C, et al: Down-regulation of microRNA-34a* in rheumatoid arthritis synovial fibroblasts promotes apoptosis resistance. Arthritis Rheum 64: 1771-1779, 2012.
30. Filková M, Jüngel A, Gay RE and Gay S: MicroRNAs in rheumatoid arthritis: potential role in diagnosis and therapy. Bio Drugs 26: 131-141,2012.

31. Kuipers H, Schnorfeil FM and Brocker T: Differentially expressed microRNAs regulate plasmacytoid vs. conventional dendritic cell development. Mol Immunol 48: 333-340, 2010.

32. Korn T, Bettelli E, Oukka M and Kuchroo VK: IL-17 and Th17 Cells. Annu Rev Immunol 27: 485-517, 2009.

33. Croft M: The TNF family in $\mathrm{T}$ cell differentiation and function - unanswered questions and future directions. Semin Immunol 26: 183-190, 2014.

34. Schenten D, Nish SA, Yu S, et al: Signaling through the adaptor molecule MyD88 in $\mathrm{CD}^{+} \mathrm{T}$ cells is required to overcome suppression by regulatory T cells. Immunity 40: 78-90, 2014.

35. Nish SA, Schenten D, Wunderlich FT, et al: T cell-intrinsic role of IL-6 signaling in primary and memory responses. Elife 3: e01949, 2014.

36. Ding L, Guo D, Homandberg GA, Buckwalter JA and Martin JA: A single blunt impact on cartilage promotes fibronectin fragmentation and upregulates cartilage degrading stromelysin-1/matrix metalloproteinase-3 in a bovine ex vivo model. J Orthop Res 32: 811-818, 2014.

37. Xue M, McKelvey K, Shen K, et al: Endogenous MMP-9 and not MMP-2 promotes rheumatoid synovial fibroblast survival, inflammation and cartilage degradation. Rheumatology (Oxford) 53: 2270-2279, 2014. 